\title{
GESTACIÓN ECTÓPICA CERVICAL TRATADA MEDIANTE PUNCIÓN ECOGUIADA INTRASACULAR DE METOTREXATO
}

\author{
Eva Lucía Tejero C., Silvia Ortega M., Belén Royo A., Pilar Andrés O., María Lapresta \\ M., José Manuel Campillos M.
}

Servicio de Obstetricia, Hospital Universitario Miguel Servet, Zaragoza, España.

\section{RESUMEN}

El embarazo cervical es una forma infrecuente de gestación ectópica. El examen ecográfico permite el diagnóstico precoz, y una actuación conservadora. Se describe el caso clínico de una gestación ectópica cervical diagnosticada en el Servicio de Urgencias de Obstetricia y Ginecología del Hospital Universitario Miguel Servet (Zaragoza, España). Paciente nulípara de 31 años que consultó por sangrado genital. El diagnóstico se realizó mediante ecografía transvaginal, objetivándose un embarazo ectópico cervical con embrión vivo acorde a 8 semanas de edad gestacional. Se realizó tratamiento conservador con metotrexato mediante inyección local intrasacular asociado a administración sistémica. Seguimiento con determinaciones seriadas de gonodotrópica coriónica humana, objetivándose un descenso progresivo de ésta. Se efectuaron controles ecográficos periódicos, evidenciando la reabsorción de la gestación confirmándose el éxito del tratamiento.

\section{PALABRAS CLAVE: Embarazo cervical, gestación ectópica, metotrexato}

\section{SUMMARY}

Cervical ectopic pregnancy is an unusual form of ectopic pregnancy. Ultrasound examination makes early diagnosis possible and permits to perform conservative treatment. We describe a case of cervical ectopic pregnancy diagnosed in the Emergency Section of Miguel Servet University Hospital (Zaragoza, Spain). A 31 year-old nullipara pregnant woman came to our Hospital because of genital bleeding. Ultrasound transvaginal examination demonstrated a cervical pregnancy with an 8 weeks embryo. Conservative treatment was prescribed using US-guided injection and systemic methotrexate. After treatment, weekly quantitative human chorionic gonadotrophin levels were determined and decreased progressively. Serial ultrasound exams were performed demonstrating how pregnancy products underwent resorption and confirming therapy success.

KEY WORDS: Cervical pregnancy, ectopic gestation, methotrexate 


\section{INTRODUCCIÓN}

El embarazo cervical es una forma inusual de presentación de la gestación ectópica. Aunque su incidencia es realmente desconocida, se estima en alrededor de 1 cada 1.000 a 18.000 embarazos, representando menos del $1 \%$ de todas las gestaciones ectópicas $(1,2)$. Esta forma de gestación ectópica se asocia a una importante morbilidad materna, derivada principalmente del sangrado producido a nivel cervical dada la incompetencia de esta estructura para cohibir la hemorragia (3), así como a un compromiso de la fertilidad futura.

El retraso en el diagnóstico del embarazo cervical contribuye al incremento de su morbilidad. Sin embargo, la sistematización de la ecografía en las consultas de obstetricia y ginecología y el desarrollo de la vía vaginal, ha permitido el diagnóstico en estadios cada vez más precoces. Esto ha provocado el uso de tratamientos más conservadores, relegando el empleo de otros más agresivos como la histerectomía, a situaciones de urgencia o fracaso terapéutico.

Numerosos tratamientos conservadores han sido descritos a fin de evitar terapias más agresivas. Las actitudes más conservadoras incluyen el empleo de metotrexato sistémico, en punción directa intrasacular o ambos simultáneamente, el cloruro potásico también en punción intrasacular, la embolización selectiva por angiografía y el legrado aspirativo bajo supervisión ecográfica seguido de taponamiento cervical con sonda de balón $(3,4,5)$.

\section{Caso clínico}

Primigesta de 31 años, sin antecedentes familiares ni médicos de interés. Acudió al Servicio de Urgencias de Maternidad del Hospital Universitario Miguel Servet de Zaragoza, España, por sangrado genital escaso como clínica prevalente en la semana 7 de gestación. En la exploración genital se objetivó un sangrado escaso procedente de cavidad uterina. El útero a la palpación estaba discretamente aumentado y los anejos parecían normales. El cérvix correspondía al de una nulípara, sin alteraciones aparentes en la especuloscopia, indoloro a la movilización. Se realizó una ecografía transvaginal en la sala de exploración de urgencias en la que se visualizó un saco gestacional intraútero localizado en tercio uterino inferior a $30 \mathrm{~mm}$ de distancia del orificio cervical externo, que contenía un embrión vivo cuya longitud cráneocaudal era acorde a siete semanas de gestación. Se visualizó también un mioma fúndico intramural de 22 mm de diámetro mayor. Ante estos hallazgos se planteó el diagnóstico diferencial entre una amenaza de aborto en una gestación intrauterina de localización baja versus una gestación ectópica cervical, por lo que se decidió nuevo control en una semana en la consulta de alto riesgo obstétrico.

La paciente acudió a consulta persistiendo únicamente la metrorragia escasa en la exploración ginecológica. Se realizó nuevamente una ecografía transvaginal, que permitió confirmar el diagnóstico definitivo de gestación ectópica cervical. Se objetivó un útero de 82 × 43 × $40 \mathrm{~mm}$, con cavidad endometrial vacía, apreciándose en tercio cervical superior, a la altura de arterias uterinas, un saco gestacional de $30 \times 27 \mathrm{~mm}$ de diámetro en cuyo interior había un embrión con latido cardiaco y acorde a 8 semanas de gestación (Figura 1 A-B). Se realizó una analítica de sangre completa junto con una determinación de la fracción beta de la hormona gonadotrópica coriónica humana (beta-HCG). El valor de esta última fue de $35.044 \mathrm{mUl} / \mathrm{ml}$, por lo que se decidió el ingreso de la paciente con el objetivo de realizar un tratamiento conservador con metotrexato.

La paciente firmó el consentimiento informado para tratamiento conservador. Se realizó en quirófano y bajo anestesia general una punción intrasacular directa transvaginal ecoguiada de metotrexato mediante aguja de $20 \mathrm{G}$. La dosis de metotrexato empleada fue de $50 \mathrm{mg}$. De forma casi simultánea, se administró metotrexato intramuscular con una dosis total de $96 \mathrm{mg}$, mediante cálculo de superficie corporal de la paciente.

Tras la comprobación de la desaparición del latido cardiaco embrionario, y la ausencia de complicaciones, la paciente fue dada de alta. Se realizó seguimiento posterior de la paciente en la consulta hospitalaria de Patología Obstétrica mediante determinaciones seriadas semanal de los niveles de la fracción beta de la gonadotropina coriónica humana y exámenes ecográficos. La cuantificación seriada de beta-HCG verificó un paulatino descenso hasta ser negativa (Figura 2).

En el primer control ecográfico realizado quince días después de la administración del metotrexato se observó la persistencia de un saco gestacional de $30 \times 27 \mathrm{~mm}$ con un embrión sin latido cardiaco, y un hematoma retrocorial con ausencia de captación Doppler. Ante estos hallazgos se decidió realizar un legrado obstétrico bajo supervisión ecográfica mediante cánula de aspiración ( $n$ ㅇ 8) y legra roma (no 4-6). Los restos obtenidos fueron enviados al Servicio de Anatomía Patológica, que confirmó el diagnóstico. 


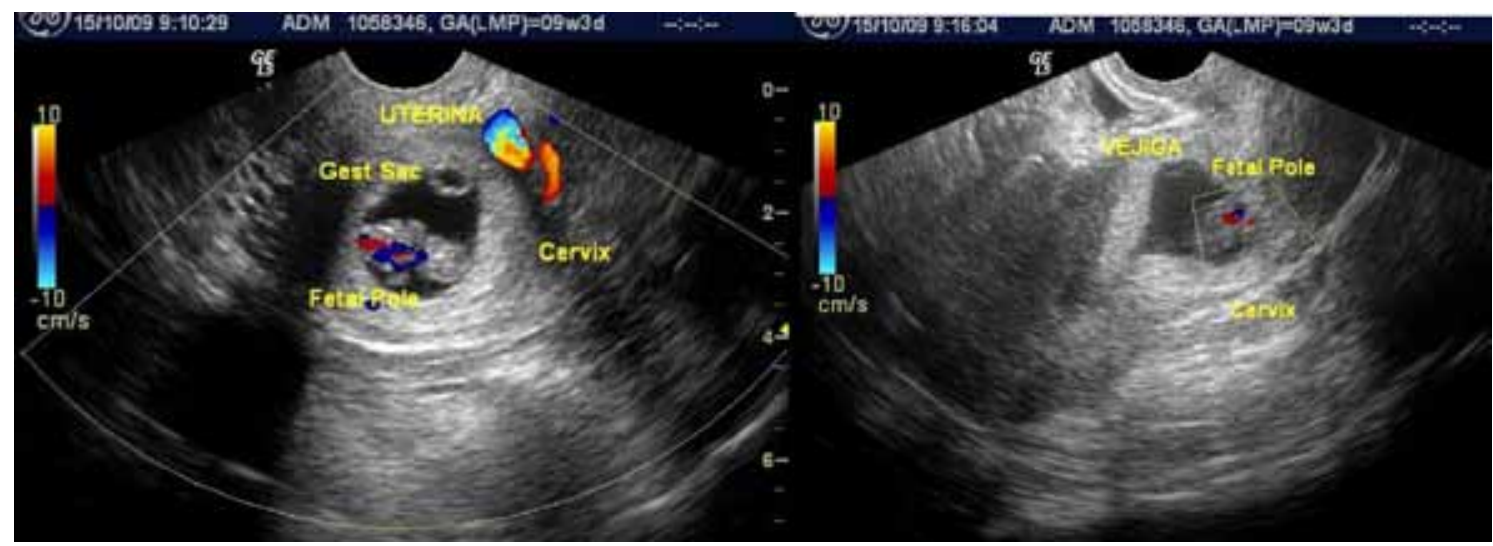

Figura 1 A-B. Gestación ectópica cervical. Cavidad endometrial vacía, apreciándose en tercio cervical superior, a la altura de arterias uterinas, un saco gestacional de $30 \times 27 \mathrm{~mm}$ con embrión vivo de 8 semanas de gestación.

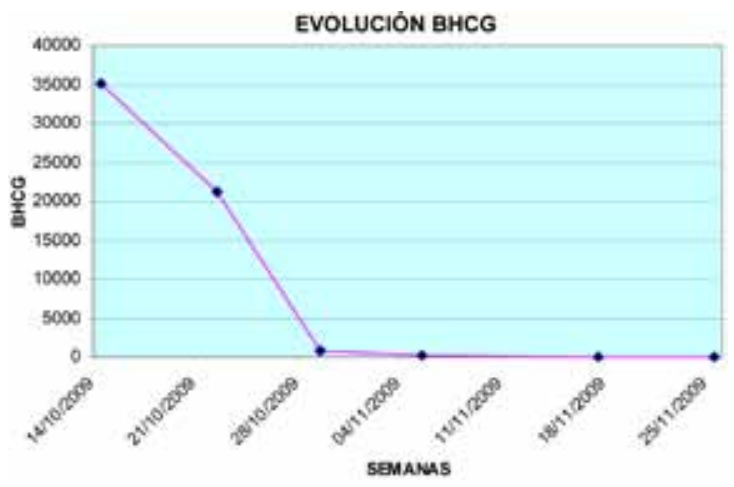

Figura 2. Evolución de subunidad beta HCG pospunción intrasacular de metotrexato.

Se continuó controlando a la paciente de forma ambulatoria, hasta obtener tres determinaciones semanales consecutivas de beta-HCG negativas. Se realizó una revisión obstétrica a los tres meses de la punción intrasacular de metotrexato. En el examen ecográfico se visualizó una imagen heterogénea a nivel cervical compatible con restos hemáticos. La paciente ya había recuperado su primer ciclo menstrual con normalidad. Cuatro semanas más tarde se realizó una nueva ecografía en la que se visualizó un cérvix de aspecto normal. Cuatro meses después del tratamiento conservador con metotrexato local y sistémico se dio el alta definitiva a la paciente.

\section{DISCUSIÓN}

La gestación localizada a nivel cervical es una forma de embarazo ectópico que puede dar lugar a un compromiso vital y reproductivo en la gestante. Afortunadamente, es una forma muy infrecuente de localización de embarazo. Algunos autores estiman su incidencia en un $1 \%$ de las gestaciones ectópicas $(2,6)$, aunque es probable que esta incidencia aumente en el futuro debido al uso cada vez mayor de las técnicas de reproducción asistida. En nuestro centro, durante el año 2009 se diagnosticaron un total de 211 gestaciones ectópicas, con un único caso a nivel cervical $(0,47 \%$ del total de gestaciones ectópicas).

Se han descrito numerosos factores de riesgo que podrían estar involucrados en el desarrollo de gestación ectópica cervical, como la presencia de cicatrices uterinas, la aplicación de técnicas de reproducción asistida, la manipulación cervical previa (dilatación con tallos de Hegar y legrado instrumental), el uso de dispositivo intrauterino o los antecedentes de enfermedad inflamatoria pélvica (7).

El desarrollo de la ecografía transvaginal como método principal de diagnóstico ha permitido detectar este tipo de gestación más precozmente. En consecuencia se están abandonando actitudes terapéuticas agresivas como la histerectomía total y se favorece un manejo más conservador de este cuadro clínico infrecuente. La utilización de la ecografía transvaginal minimiza la distancia desde la sonda hasta el cervix, mejorando la calidad de la imagen y sus detalles. Este tipo de ecografía resulta más útil a la hora de establecer el diagnóstico diferencial entre gestación cervical y gestación intrauterina que la ecografía transabdominal. Además, la utilización del Doppler color posibilita establecer la distinción entre una gestación ectópica cervical viable y la presencia únicamente de restos abortivos, debido a la diferencia de aporte sanguíneo que existe entre ambas 
entidades (2).

El metotrexato es un agente quimioterápico que ejerce su mecanismo de acción a través de la unión con la enzima dihidrofolato reductasa, la cual participa en la síntesis de nucleótidos de purinas. Esto interfiere con la síntesis del ADN y perturba la multiplicación de células. Su eficacia sobre el tejido trofoblástico ha sido bien establecida.

La primera vez que se describió el uso de metotrexato como tratamiento médico sistémico en obstetricia, fue en 1982, en un caso de gestación ectópica localizada a nivel tubárico (8). Desde entonces se han propuesto múltiples protocolos acerca de su uso, incluyendo los casos de gestación ectópica cervical mediante inyección intrasacular del mismo. En 1989 Palti y cols (9), describieron el uso intracervical de metotrexato, seguido de una inyección vía intramuscular; Kaplan y cols (10) y Timor-Tristch y cols (11) comunicaron también su uso intrasacular bajo supervisión ecográfica, todos con resultados favorable.

La punción intrasacular de $50 \mathrm{mg}$ de metotrexato es un método óptimo para la resolución conservadora de este cuadro. En el caso descrito se asoció además una dosis única de metotrexato sistémico calculada según peso y talla de la paciente. Los principales efectos secundarios derivados del uso de metotrexato son: gastritis, estomatitis, nauseas y alteraciones analíticas como elevación de las transaminasas hepáticas, leucopenia o trombocitopenia. No obstante son poco frecuentes, pudiendo afirmarse que el tratamiento suele ser bien tolerado por la mayoría de las pacientes (12).

\section{CONCLUSIÓN}

Se destaca la relevancia de un correcto control postratamiento, basado en la determinación periódica de los niveles de beta-HCG y en la realización de exámenes ecográficos seriados. La resolución completa del cuadro y el éxito del tratamiento sólo podrá confirmarse cuando se negativicen los niveles de beta-HCG y la estructura ecográfica cervical se encuentre dentro de la normalidad.

\section{BIBLIOGRAFÍA}

1. Troncoso F, Cardone X, Rondini C, Troncoso C. Embarazo ectópico cervical. Diagnóstico y tratamiento conservador con cerclaje cervical. Rev Chil Obstet Ginecol 2005;70(4):257-60.

2. Plascencia N, Hernández MP, Guadarrama R, Guerra L, Salmón JFG, Galván A. Embarazo cervical. Tres casos. Ginecol Obstet Mex 2008;76(12):744-8.

3. Hassiakos D, Bakas P, Creatsas G. Cervical pregnancy treated with transvaginal ultrasound-guided intraamniotic instillation of methotrexate. Arch Gynecol Obstet 2005;271(1):69-72.

4. Has R, Balci NC, Ibrahinoglu L, Rozanes I, Topuz S. Uterine artery embolization in a 10 week cervical pregnancy with coexisting fibroids. Int J Gynecol Obstet 2001;72(3):253-8.

5. Fylstra DL, Coffey MD. Treatment of cervical pregnancy with cerclage, curettage and balloon tamponade. A report of three cases. J Reprod Med 2001;46(1):71-4.

6. Celik C, Bala A, Gezginc K, Akyurek C. Methotrexate for cervical pregnancy. A case report. J Reprod Med 2003;48(2):130-2.

7. Ginsburg ES, Frates MC, Rein MS, Fox JH, Hornstein MD, Friedman AJ. Early diagnosis a treatment of cervical pregnancy in an invitro fertilization program. Fertil Steril 1994. 61(5):966-999.

8. Potter M, Lepine L, Jamieson DJ. Predictors of success with Methotrexate treatment of tubal ectopic pregnancy at Grady Memorial Hospital. Am J Obstet Gynecol 2003; 188(5): 1192-4.

9. Palti Z, Rosenn B, Goshen R, Ben-Chitrit A, Yagel S. Successful treatment of a viable cervical pregnancy with methotrexate. Am J Obstet Gynecol 1989;161(5):1147-8.

10. Kaplan BR, Brandt T, Javaheri G, Scommegna A. Successful treatment of a live cervical pregnancy with methotrexate and folinic acid. A case report. J Reprod Med 1989;34(10):853-6.

11. Timor-Tristch I.E, Monteagudo A, Mandeville EO, Peisner DB, Anaya GP, Pirrone EC. Succesful management of viable cervical pregnancy by local injection of methotrexate guided by transvaginal ultrasonography. Am J Obstet Gynecol 1994;170(3):737-9.

12. Stovall TG, Ling FW, Gray LA. Single-dose methotrexate for treatment of ectopic pregnancy. Obstet Gynecol 1991;77(5):754-7. 